Received: March 22, 2021

Accepted: March 21, 2021

\section{Correspondence}

Carlo Salomone

Infectious Diseases and Septic Orthopaedic Unit (MIOS), Santa Maria di Misericordia Hospital,

Albenga, Savona, Italy

E-mail: salomone.c@libero.it

\section{Conflict of interest}

The authors have no conflict of interest to declare.

How to cite this article: Salomone C, Martínez-Herreros I, Formica M, et al. Circular external fixation: the method. Lo Scalpello Journal 2021;35:53-56. https://doi.org/10.36149/03905276-206

(C) Ortopedici Traumatologi Ospedalieri d'Italia (O.T.O.D.I.) 2021

\section{(c) (i) () $\Theta$}

This is an open access article distributed in accordance with the CC-BY-NC-ND (Creative Commons Attribution-NonCommercial-NoDerivatives 4.0 International) license. The article can be used by giving appropriate credit and mentioning the license, but only for non-commercial purposes and only in the original version. For further information: https://creativecommons.org/licenses/by-nc-nd/4.0/deed.en

\title{
Circular external fixation: the method
}

\author{
Carlo Salomone', Isabel Martínez-Herreros ${ }^{2}$, Matteo Formica², \\ Federici Marcello ${ }^{3}$, Stefano Tornago ${ }^{4}$, Andrea Antonini', \\ Giuliana Carrega', Giovanni Riccio' \\ ${ }^{1}$ Infectious Diseases and Septic Orthopaedic Unit (MIOS), Santa Maria di Misericordia \\ Hospital, Albenga, Savona, Italy; ${ }^{2}$ Orthopaedic Clinic, IRCCS Policlinico San \\ Martino University Hospital, Genoa, Italy; ${ }^{3}$ Department of Orthopaedic Surgery and \\ Traumatology, Santa Corona Hospital, Pietra Ligure, Savona, Italy; ${ }^{4}$ Department of \\ Prosthetic Surgery, Santa Corona Hospital, Pietra Ligure, Savona, Italy
}

\begin{abstract}
SUMMARY
In this study, indications and characteristics of the system are illustrated by using the experience of a reference center for the treatment of osteoarticular diseases with circular external fixation. Deeper insights of the method and the technique of implantation into biomechanics by using circular external fixation will be provided step by step. For simplicity and due to the frequency of system application, we will refer to diaphyseal systems of long bones such as the tibia.

Compliance with biological principles as low aggressiveness, mechanical principles as adjustable mechanics with possibility of interaction throughout the whole period of treatment, from exterior to interior of the fixation system, following a safe anatomical path and implantation wire technique, indications in traumatology (high energy, comminuted, open and with loss of substance fractures) and sequelae (non-union, osteomyelitis and deformation) allow a good tolerance of the system and proper achievement of results while minimizing surgical complications and risks ${ }^{1-3}$.

The use of different instruments, also including newly developed ones, does not alter the fundamental principles of the method.
\end{abstract}

Key words: circular external fixation, method, tibia fracture, non-union, osteomyelitis

\section{Introduction}

Circular external fixation has always been mostly considered as a limb salvage technique in compromised bone and limb (soft tissue and vascularization) situations ${ }^{4}$. These indications are satisfied by the bone recovery potential through distraction osteogenesis, deformity correction, and the use of wire with low infectious risk for the management of osteomyelitis and postoperative infections. In fact, external fixation must be considered as a method of osteosynthesis as well as treatment of complications. It is a correct and valid method as the internal synthesis with nails or plates and screws ${ }^{4}$.

External fixators are different instruments of a single method, which must be applied according to their characteristics. Application according to the method of circular external fixation allows it to take full advantage and minimize risks and complications. Furthermore, the appearance of the latest generation instruments, external fixators with greater attention to hardware and always implanted according to the method, allow a facilitated and more satisfactory approach to the application of the system. 
In this short manuscript we will focus on the individual stages of application and the fundamental concepts of applying circular external fixation. Starting from the positioning of the wire, their stabilization and connection on the exoskeleton, eventual reduction maneuvers and finally the definitive stabilization of the system, with special attention to always respect the biomechanical balance, which is the basis of this method.

To create the best environment and biomechanical treatment to lead the patient to recovery there should be: respect for biology (low aggressive application of the system) and consequent respect for vascularization and soft and bone tissues, the execution of a mechanically stable but dynamizable implant over time utilizing the intrinsic potential of circular external fixation systems while always respecting the method and anatomy ${ }^{5,6}$. The work aims to describe step by step a circular external fixation implant, for greater simplicity and frequency of implantation, we have considered a tibial bone lesion site, with the goal of giving the reader the appropriate and necessary knowledge to respect the method of application of a circular external fixator ${ }^{5}$.

\section{Materials and methods}

In cases of fracture focus or complications (such as pseudarthrosis or after radical bone and soft tissue debridement for osteomyelitis) of diaphyseal tibia, the use of circular external fixation is indicated in cases of high energy trauma, soft tissue destruction, multi-fragmentation, long oblique rhyme, infection, and loss of substance. We proceed to describe how to implant the external fixator and how to perform the reduction and stabilization necessary to create a biomechanical environment that leads to patient healing ${ }^{7}$.

This description aims to clarify and spread the knowledge of the circular external fixation method which, based on precisely biomechanical principles, must first of all respect the biology of the site to be treated, avoiding, for example, opening a fracture site and devascularizing the bone with excessively bloody traumatic acts, but taking advantage of the physiological laboratory formed by the hematoma, which is rich in growth factors ${ }^{8}$.

In fact, it is emphasized that external fixation leads to consolidation by second intention, and therefore with hypertrophic bridging callus, and not as an internal reduction where the synthesis has the objective of perfectly anatomical reconstruction and consequent healing by first intention ${ }^{9}$.

Thus, the first step to consider is the application of the two threads of the circular external fixator proximal and distal respectively, as the traction mechanism of the fracture focus with control of rotations and realignment of the mechanical axis.

\section{Role of the first and second wires}

All the tract (pin or $\mathrm{k}$ wire) must be applied and positioned in the respect of the safety corridors. The first wire to be positioned is the proximal one (always referring to the tibia), parallel to the joint line of the knee in anterior-posterior and laterally. The second distal wire is positioned parallel to the tibio-tarsal joint in anterior-posterior and lateral. Next, an X-ray control in the operating room will be done.

Wire connection to the system takes place by locking the exoskeleton on one side and and the consequent contralateral traction according to the methodology (110-130 Newton on corticals, 90 Newton on cancellous bone and for threads with olive, except for special situations).

We start by blocking the proximal thread, paying particular attention to center the limb on the circular exoskeleton, avoiding excessive distance which would reduce mechanical stability and annoying decubitus, especially in the posterior compartment of the tibia.

The second wire to be stabilized and tensioned is the distal one, which helps center the limb inside the exoskeleton and stabilize the rotations of the lower limb (Fig. 1). Therefore, in the case of a tibia it is essential to pay particular attention to the alignment of the patellar tuberosity with the level of the second metatarsal foot.

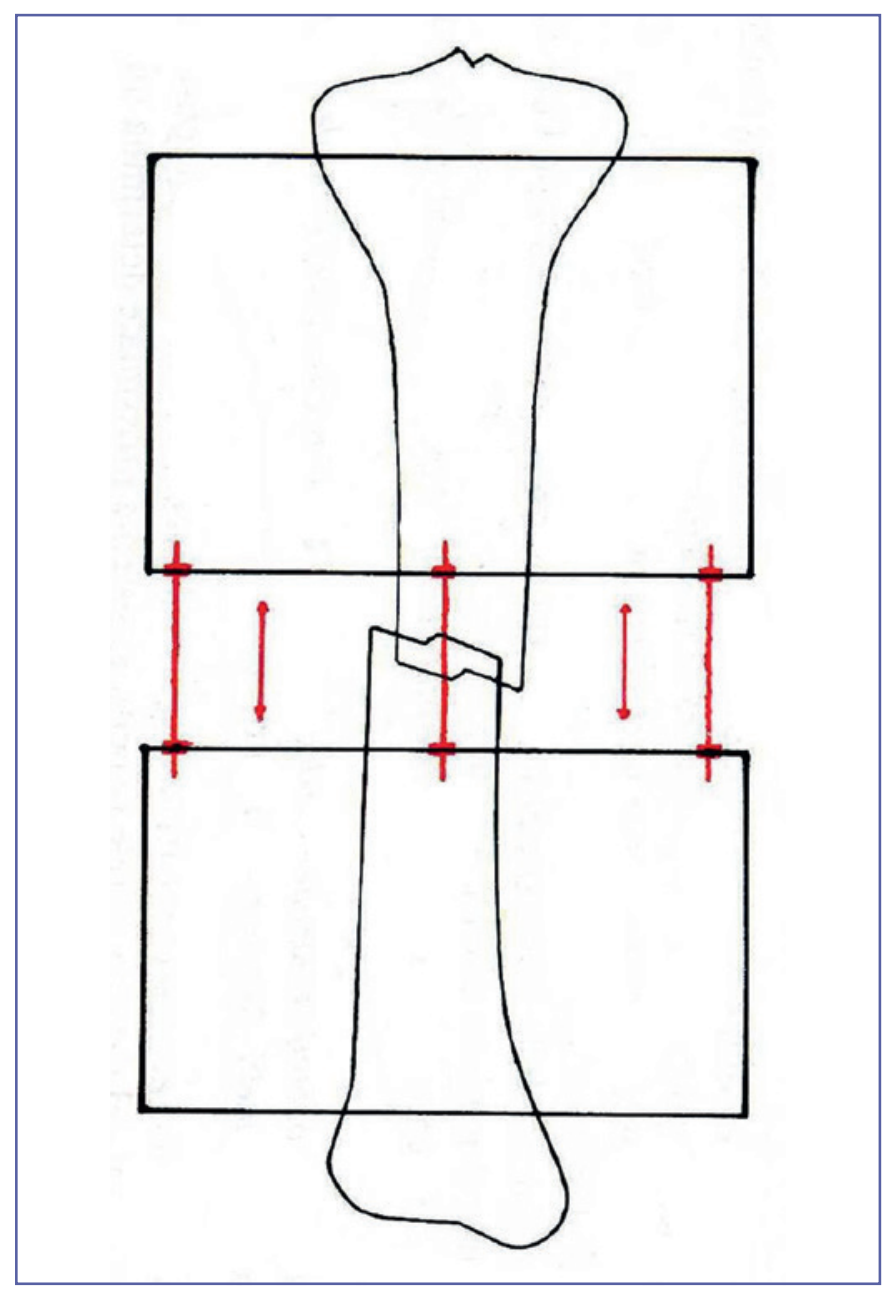

Figure 1. Proximal and distal $\mathrm{K}$ wire. 
Once these first two stabilization points have been performed (for example for a fracture focus), we can distract the central region of the system, where bars of sufficient length must have been prepared to achieve the necessary millimeters of distraction, or in the case of new systems, the use of telescopic bars (extendable) allow more simply and without the need to take measurements, to perform the distraction quickly. Furthermore, these bars permit the connection even with slight inclinations on the axis, thus allowing a correct restoration of the mechanical load axis of the tibia without the need of assistive devices (such as hings or washer) ${ }^{10,11}$.

\section{Axis correction}

The next step, if needed, is to proced with corrections, controlling the focus of the fracture with fluoroscopy.

On the antero-posterior plane, we proced to correct the axis varus-valgus by applying threads with k-olive stopper-stabilizator or corrector that must be pulled using the transport that the olive performs on the tibial stump before being blocked on the ring against the contralateral side (Fig. 2).

Once this correction is satisfactory, we proceed with closure in the olive site and tensioning of the wire (90 Newton). The traction is performed on the side towards which we want to obtain the reduction (on the opposite site to the deformity), while the olive will be applied and supported on the deformity side.

It is not always necessary to do corrections, but it minimizes the risk of secondary scomposition-dislocation in the fracture, pseudoarthrosis or elongation site.

In the same way, it is possible to make corrections on the lateral plane, making procurvatum or recurvatum, using the concepts of hammok wires, i.e., wires must be blocked using the holes at a level below or above the wire exit according to the need of eventual corrections. To produce a procorvatum it will be necessary to block the wire using the holes at the level below to perform a correction movement in the direction of the recurvatum need for correction the procurvatum (opposite reasoning and execution for correcting a recurvatum) (Fig. 3).

The correct use of this method allows a gradual and progressive repositioning of the tibial axis laterally during the tensioning of the wires ${ }^{11}$.

\section{System stabilization}

Once made the corrections on the two planes and reached a satisfactory reduction or a correct position of the tibial stumps to heal by secondary intention, it is sufficient to proceed with stabilization of the system using wires or screws.

The use of screws permit good tolerance and good stability, especially at the margins of the system, and is indicated render the the fracture site biomechanically more elastic for support dinamization with the cyclic micromovement activated from the wheight bearing.

The connection areas of the pin and k-wire tract must be distributed on two levels, respectively, in the proximal part and

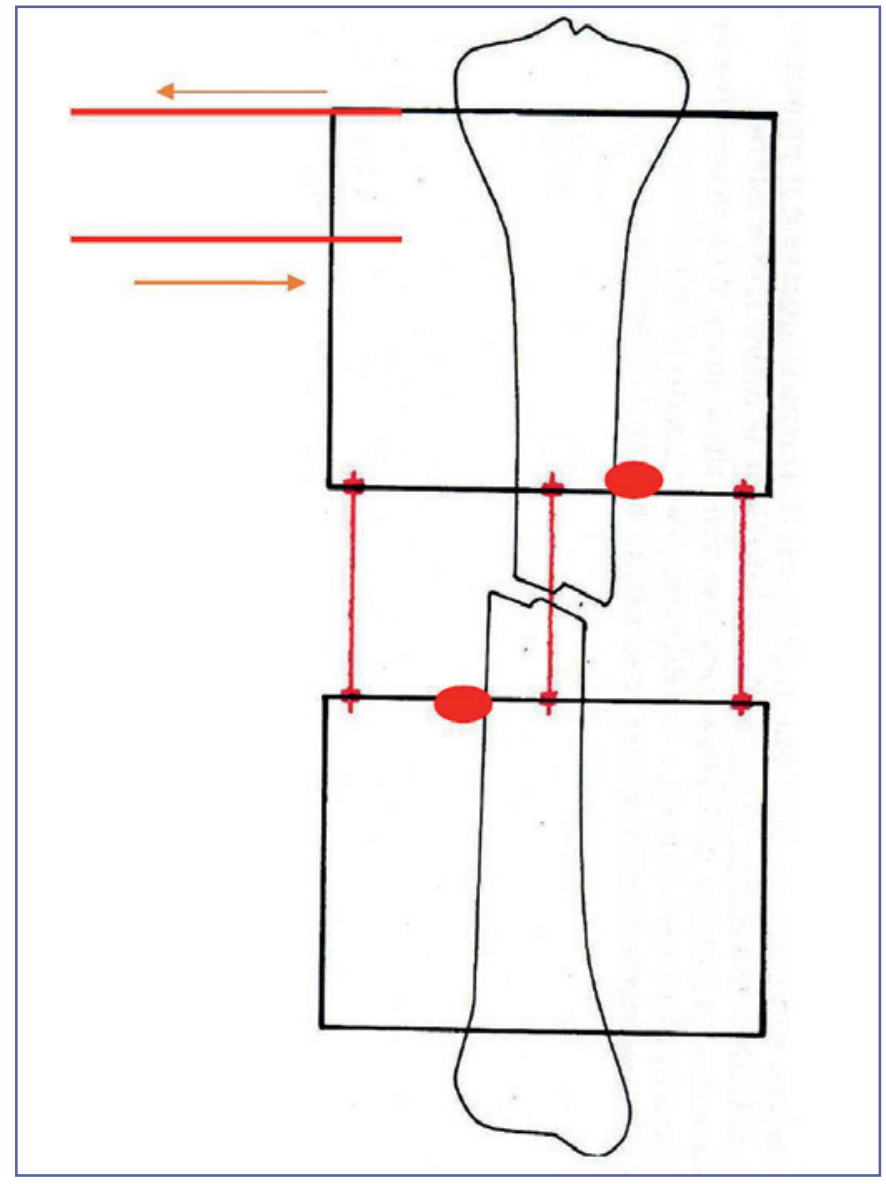

Figure 2. $\mathrm{K}$ olive wire for reduction in anteroposterior view.

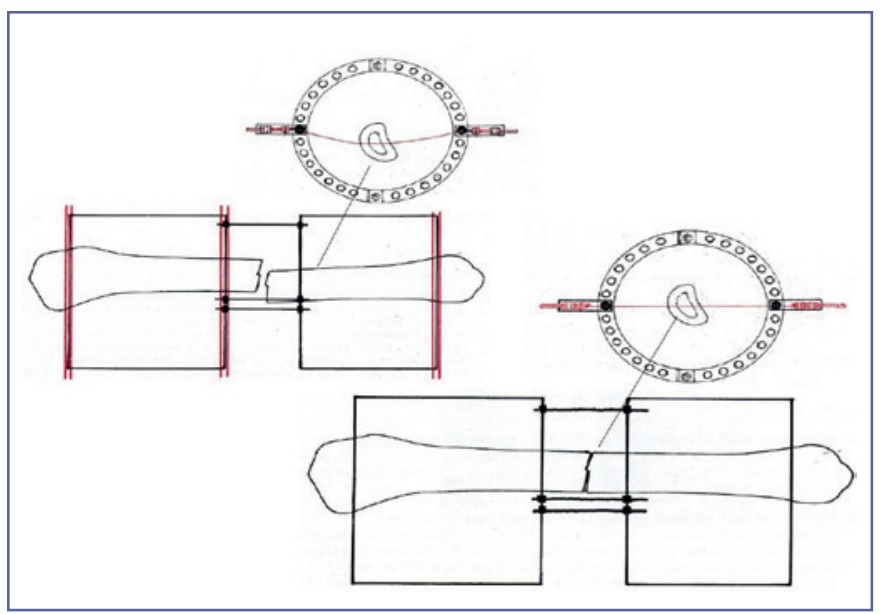

Figure 3. Hammok $\mathrm{K}$ wire for reduction in lateral view.

in the distal part of the treatment site. It has to be positioned using the extension of the available in the proximal and distal tibial stumps in order to create a lever arm that is more stable 
for movements on the treatment site. The more stable tract application in the different site of the lever arm for the two tibial stumps, are perpendicular to gravity.

The wrong use of a single grip area, i.e. a single ring (without applying multi hole plate at different level) could create greater elasticity and instability resulting in pain, destruction of the wires, and a negative evolution of the treatment ${ }^{11}$.

The description of the method presented above could have different interpretations and could also be applied according to other criteria (e.g. in the application of a external fixator on a limb in trans-skeletal traction, the external fixator is exclusively a stabilizing element, because the reduction has already been made.) It allows to build a scheme-protocol for the application of the system ${ }^{12}$.

The use of tools in which the hardware is more accurate, fast and reliable, reduce surgery time, reduces fluoroscopy time, and makes for easier application of the system.

\section{Results}

The use of external fixation in traumatology and in the experience based in cases in our orthopedic surgery center of reference according to the method has made it possible to reduce surgical application time associating increasingly accurate reduction results and also reduce intraoperative fluoroscopy times. The implanted systems showed greater tolerance $7,8,10,11$. The evolution of hardware in terms of materials, accuracy and finish has been of considerable help and has allowed an increasingly accessible and reliable application.

\section{Discussion}

Considered as a technique that achieves consolidation by second intention and takes advantage of the unique potential of regeneration, circular external fixation has shown itself to be an irreplaceable role in orthopedics and traumatology when applied in compliance with the principles and concepts of biomechanics ${ }^{2,3,5,6}$. The results are strictly dependent on compliance with the method even if supported by the evolution of the instruments available on the market.

The objective and dissemination of a method described in this way aims to create a network of users who speak the same language and can deal with a working pattern based not only on empiricism, but on the reproducibility of methodology, necessarily with variants according to the innovation and general and local situations of the patient and the type of instrument utilized.
We must not overlook how important dedication is in following in time the systems and the long learning curve that the method of circular external fixation requires $4,7,8,10$.

\section{Conclusions}

The evolution of the system, intended as the circular external fixator, has made the application of an implant more dynamic and simple, and the bio-mechanical characteristics and the implant methodology must be known in depth through a long learning of the principles of the technique. Circular external fixation is still considered a methodology used by a select few and exclusively restricted to indications for desperate treatments of limb salvage, but it can be recognized as a method of osteosynthesis and treatment in the orthopedic and traumatologic field ${ }^{4}$.

The effort by companies who produce this systems to make it more accessible allows greater potential for surgeons to be able to recognize the role of circular external fixation.

\section{References}

1 Castaman E. Osteogenesi ripartiva: strategia dei movimenti. Villafranca Padovana: Runa Editrice 2011.

2 Charnley J. Trattamento incruento delle fratture comuni. Padova: Piccin 1984.

3 Bossi E, Azioni R, Riva R, et al. Osteosintesi esterna: indicazioni e tecniche nella traumatologia dell' apparato locomotore. Milano: Masson 1983.

4 Biasibetti A. La fissazione esterna: un metodo. I fissatori esterni: strumenti di un metodo. Atti congressuali 1988.

5 Bianchi-Maiocchi A, Marti Gonzalez JC. Osteosintesis: tecnica de Ilizarov. Las Rozas, ES: Ediciones Norma 1989.

6 Castaman E. Principi biomeccanici di fissazione esterna. Bologna: Aulo Gaggi Editore 1983.

7 Salomone C, Burastero G, Rita A, et al. Le pseudoartrosi nelle fratture diafisarie. Lo Scalpello 2015;29:44-48. https://doi. org/10.1007/s11639-015-0102-3

8 Biasibetti A, Aloj D, Di Gregorio G, et al. Mechanical and biological treatment of long bone non-unions. Injury 2005;36(Suppl 4):S45-50. https://doi.org/10.1016/j.injury.2005.10.013

9 Sarmiento A, Latta LL. Closed functional treatment of fractures. New York, NY: Springer 1981.

10 Salomone C, Busastero G, Rita A, et al. Le infezioni nelle fratture diafisarie. Lo Scalpello 2015;29:49-54. https://doi.org/10.1007/ s11639-015-0100-5

11 Bianchi-Maiocchi A, Catagni M. Osteosintesi transossea nelle fratture di gamba. Bollettino ASAMI 1985.

12 Catagni M. Atlas for the insertion of transosseous wires and halfpins (Ilizarov method). Editor Bianchi-Maiocchi 2002. 